\title{
Continuous Space Estimation for WLAN Location Determination Systems
}

\author{
Moustafa Youssef, Ashok Agrawala \\ Department of Computer Science \\ University of Maryland at College Park \\ Email:\{moustafa, agrawala\}@cs.umd.edu
}

\begin{abstract}
WLAN location determination systems add to the value of a wireless network by providing the user location without using any extra hardware. Current systems return the estimated user location from a set of discrete locations in the area of interest, which limits the accuracy of such systems to how far apart the selected points are. In this paper, we present two techniques to estimate the user location in the continuous physical space, namely the Center of Mass technique and Time Averaging technique. We test the performance of the two techniques in the context of the Horus WLAN location determination system under two different testbeds. Using the Center of Mass technique, the performance of the Horus system is enhanced by more than $13 \%$ for the first testbed and more than $6 \%$ for the second testbed. The TimeAveraging technique enhances the performance of the Horus system by more than $24 \%$ for the first testbed and more than $15 \%$ for the second testbed. The techniques are general and can be applied to any of the current WLAN location determination systems to enhance their accuracy. Moreover, the two techniques are independent and can be applied together to further enhance the accuracy of the current WLAN location determination systems.
\end{abstract}

Index Terms - location-aware systems, location determination architecture, continuous space estimation, user positioning, wireless LAN.

\section{INTRODUCTION}

WLAN location determination systems have been an active area of research for the latest years [1-17]. WLAN location determination systems add to the value of the wireless networks by providing the user location without using any extra hardware. This enables a set of context-aware applications [18] on top of the wireless networks such as location-sensitive content delivery, direction finding, asset tracking, and emergency notification.

WLAN location determination systems usually work in two phases: offline training phase and online location determination phase. During the offline phase, the signal strength received from the access points at selected locations in the area of interest is tabulated, resulting in a so-called radio map. During the location determination phase, the signal strength samples received from the access points are used to "search" the radio map to estimate the user location. One of the discrete radio map locations is returned as the estimated location.

In this paper, present two techniques for estimation the user location in the continuous user space removing the constraints of the discrete location estimation. The first technique, the Center-of-Mass technique, estimates the user location based on the list of candidate locations in the current estimation period. The second technique, the Time-Averaging technique, estimates the user location using the history of consecutive estimates.

We implement the two technique in the context of the Horus WLAN location determination system [1-5], which is a probabilistic WLAN location determination system. We also test the system under two different testbeds and discuss how to combine the two techniques together to further enhance the accuracy of WLAN location determination systems.

The rest of the paper is organized as the following. Section II briefly describes the Horus system. In Sections III and IV we present the details of the Center-of-Mass technique and the Time-Averaging technique respectively. We present the evaluation of the two techniques in Section V. Finally, we discuss related work in Section VI and conclude the paper in Section VII.

\section{The Horus SySTEM}

In this section, we present a brief overview of the Horus system [1-5]. Our goal is to provide context for the techniques presented in rest of the paper. Horus is a probabilistic location determination system. The main goal of the system is to identify the noisy characteristic of the wireless channel and to develop techniques to handle them. Figure 1 shows the components of the Horus system. The system uses the signal strength information returned from different access points to infer the user location and to provide an API for the user applications to use the system functionality.

The system works in two phases:

1) Offline phase: to build the radio map, cluster radio map locations, and do other preprocessing of the signal strength models.

2) Online Phase: to estimate the user location based on the received signal strength from each access point and the radio map prepared in the offline phase.

The radio map stores the distribution of signal strength received from each access point at each location. There are two modes for operation of the Horus system: one uses nonparametric distributions and the other uses parametric distributions. In this paper, we will use the parametric distribution mode in which the signal strength distributions are modeled using Gaussian distributions.

The Clustering module is used to group radio map locations based on the access points covering them. Clustering is used 
to reduce the computational requirements of the system and, hence, conserve power.

The Discrete Space Estimator module returns the radio map location that has the maximum probability given the received signal strength vector from different access points. An outline of the algorithm used is given in Algorithm 1.

The Small-Scale Compensator module handles the smallscale variation characteristics of the wireless channel [3].

The Continuous Space Estimator takes as an input the discrete estimated user location, one of the radio map locations, and returns a more accurate estimate the user location in the continuous space. This module is the subject of this paper.

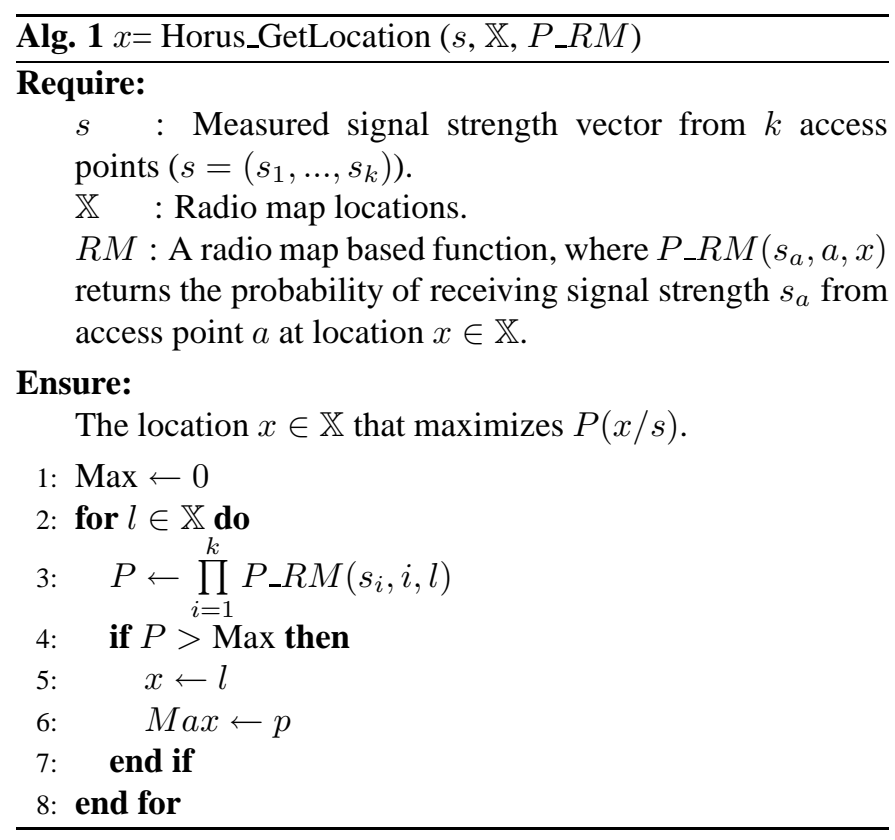

\section{Center of Mass Technique}

The Center of Mass technique is based on treating each location in the radio map as an object in the physical space whose weight is equal to the normalized probability ${ }^{1}$ assigned by the discrete-space estimation process. We then obtain the Center of Mass of the $N$ objects with the largest mass, where $N$ is a parameter to the system.

More formally, let $p(x)$ be the probability of a location $x \in \mathbb{X}$, i.e. the radio map, and let $\overline{\mathbb{X}}$ be the set of locations in the radio map ordered in a descending order according to the normalized probability. The Center of Mass technique estimates the current location $x$ as:

$$
x=\frac{\sum_{i=1}^{\min (N,\|\overline{\mathbb{X}}\|)} p(i) * \overline{\mathbb{X}}(i)}{\sum_{i=1}^{\min (N,\|\overline{\mathbb{X}}\|)} p(i)}
$$

where $\overline{\mathbb{X}}(i)$ is the $i^{t h}$ element of $\overline{\mathbb{X}}$

Note that the estimated location $x$ need not to be one of the radio map locations. Algorithm 2 shows the details of the Center of Mass technique.

\footnotetext{
${ }^{1}$ The normalization is used to ensure that the sum of the probabilities of all locations equals one.
}

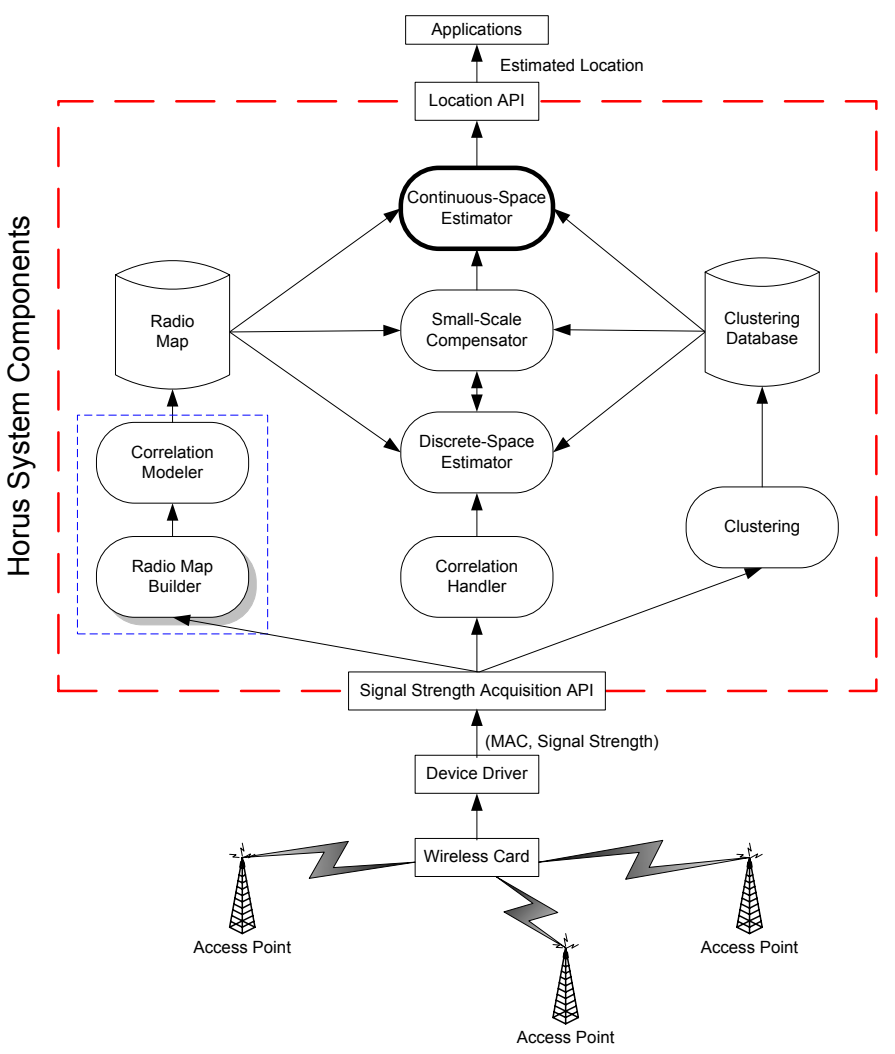

Fig. 1. Horus Components: the arrows show information flow in the system. Shaded block represent modules used during the offline phase. In this paper, we describe the continuous space estimator component of the Horus system (shown in thick lines).

Intuitively, the higher the probability of a location, the closer the estimated position should be to this location. For example, consider the case in Figure 2. The figure shows the simple case where the radio map consists of two locations: $A$ and $B$. Assume that the user is standing in the middle between the two locations. In this case, the signal strength vector obtained from this location will be different from the signal strength vector expected at either location $A$ or location $B$. Let's assume that for the given signal strength vector the discrete-space estimation process assigns a normalized probability of 0.55 for location $A$ and 0.45 for location $B$. In this case, the discrete-space estimation process will return location $A$ as the estimated location. However, the technique proposed in this section would return a location estimate in the middle of the two locations, slightly biased towards location $A$, which should be a more accurate estimate to the actual location than location $A$. We study the performance of this technique compared to the discrete-space estimator in Section V.

\section{Time Averaging Technique}

The Time Averaging technique uses a time-average window to smooth the resulting location estimate. The technique obtains the location estimate by averaging the last $W$ locations estimates obtained by the discrete-space estimator.

More formally, given a stream of location estimates $x_{1}, x_{2}, \ldots, x_{t}$, the technique estimates the current location $\overline{x_{t}}$ 


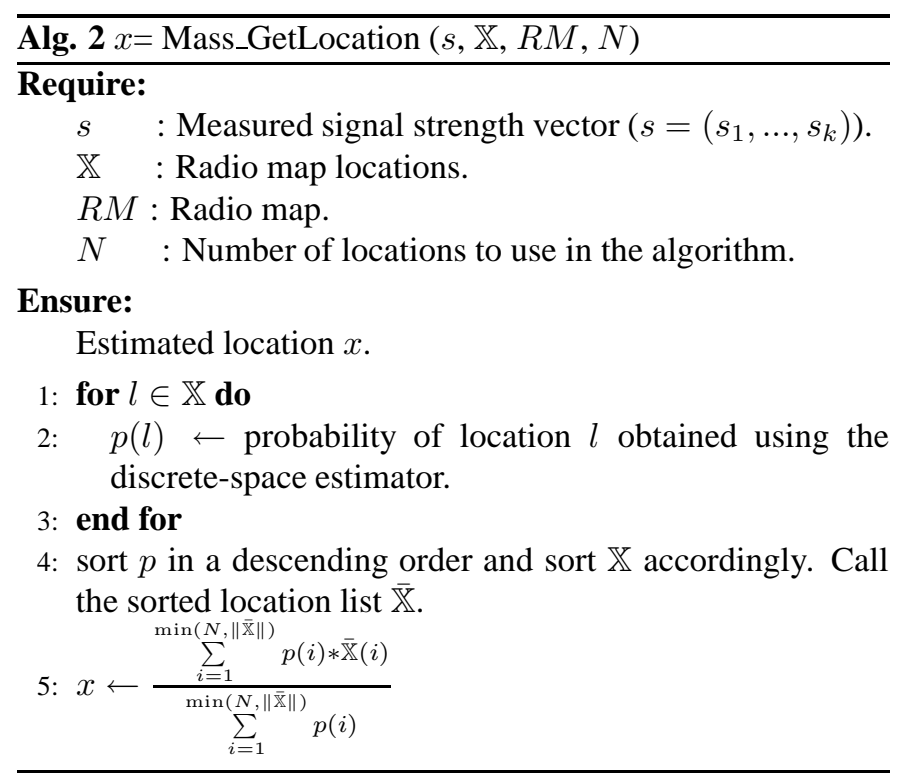

\section{Estimated location using the Center of Mass technique}

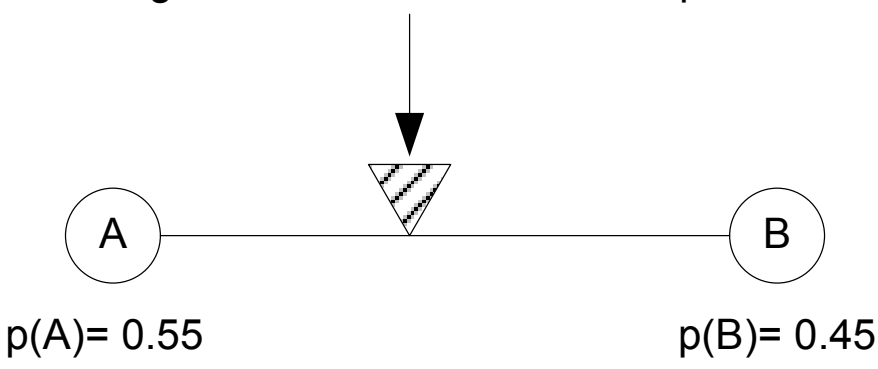

Fig. 2. An example of the Center of Mass technique. Here location $A$ has probability of 0.55 and location $b$ has a probability of 0.45 . The Center of Mass technique will return the estimated location in the middle of the two locations, slightly biased to location $A$. The discrete-space estimator will return location $A$ as the location estimate.

at time $t$ as:

$$
\bar{x}_{t}=\frac{1}{\min (W, t)} \cdot \sum_{t-\min (W, t)+1}^{t} x_{i}
$$

Algorithm 3 shows the details of the time-averaging technique.

\section{EXPERIMENTAL EVALUATION}

In this section, we discuss the experimental testbeds and evaluate the performance of the Center of Mass and Time Averaging techniques comparing them to previous work in the area of WLAN location determination.

\section{A. Experimental Testbeds}

In this section, we present the experimental testbeds we used to evaluate the performance of the Horus system and compare its performance to other systems.

1) Signal Strength Acquisition: Using the device driver and the API we developed [19], we collected 200 samples at each location, one sample per 100 milliseconds. The cards used were Lucent Orinoco silver NICs supporting up to $11 \mathrm{Mbit} / \mathrm{s}$ data rate [20]. To test the performance of the system, we used an

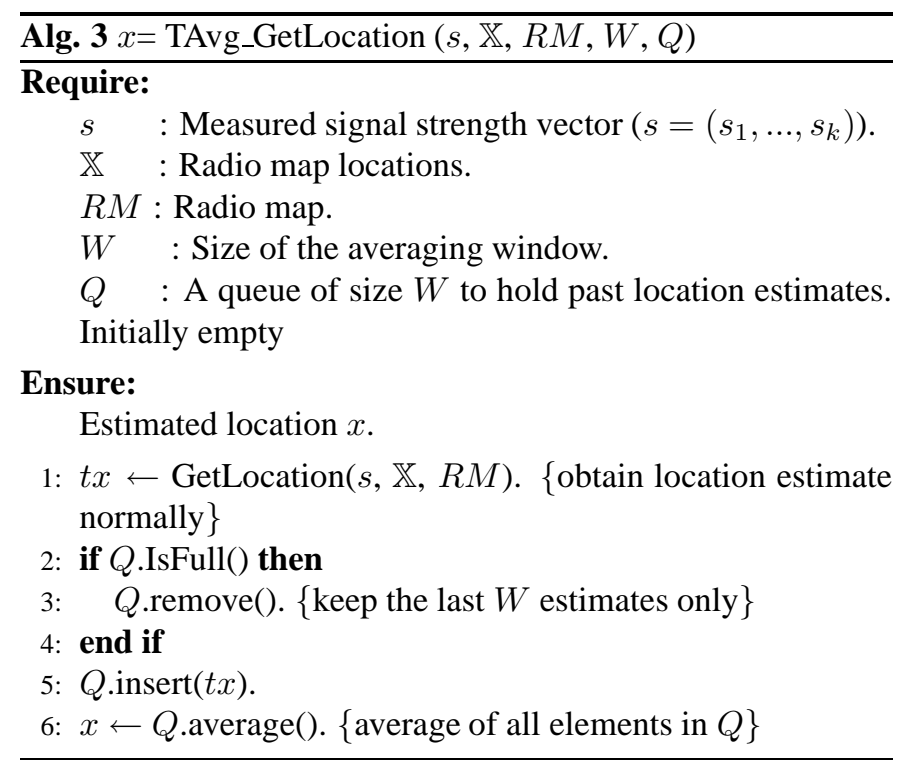

independent test set that was collected on different days, time of day, and by different persons than the training set.

2) Testbed 1: We performed our first experiment in the south wing of the fourth floor of the A. V. William's building in the University of Maryland at College Park. The layout of the floor is shown in Figure 3. The wing has a dimension of 224 feet by 85.1 feet. The technique was tested in the University of Maryland wireless network using Cisco access points ${ }^{2}$. The entire wing is covered by 21 access points.

The radio map has 110 locations along the corridors and 62 locations inside the rooms. On the average, each location is covered by 6 access points. The test set was collected by different persons on different days and time of days. The Horus system was running on Windows $X P$ professional operating system.

3) Testbed 2: We performed the second experiment in the an office space (Figure 4). The area of the experiment site is approximately 39 feet by 118 feet covering corridors, cubicles, and rooms. The test area was covered by 6 access points. Five of the 6 access points were LinkSys and the remaining one was a Cisco access point.

We have a total of 110 locations in the radio map. On the average, each location is covered by 4 access points. The test set was collected by different persons on different days and time of days. The Horus system was running under Linux (kernel 2.5.7) operating system.

\section{B. Results}

In this section, we compare the performance of the two techniques for obtaining a continuous-space location estimate.

1) Center of Mass Technique: Figures 5 and 6 show the effect of increasing the parameter $N$ (number of locations to interpolate between) on the performance of the Center of Mass technique for the two testbeds. Note that the special case of $N=1$ is equivalent to the discrete-space estimator output. The figures show that the performance of the Horus system is enhanced by more than $13 \%$ for the first testbed and more than $6 \%$ for the second testbed for $N=6$.

\footnotetext{
${ }^{2}$ This testbed is different from the initial testbed we used in our previous work [1-4]
} 


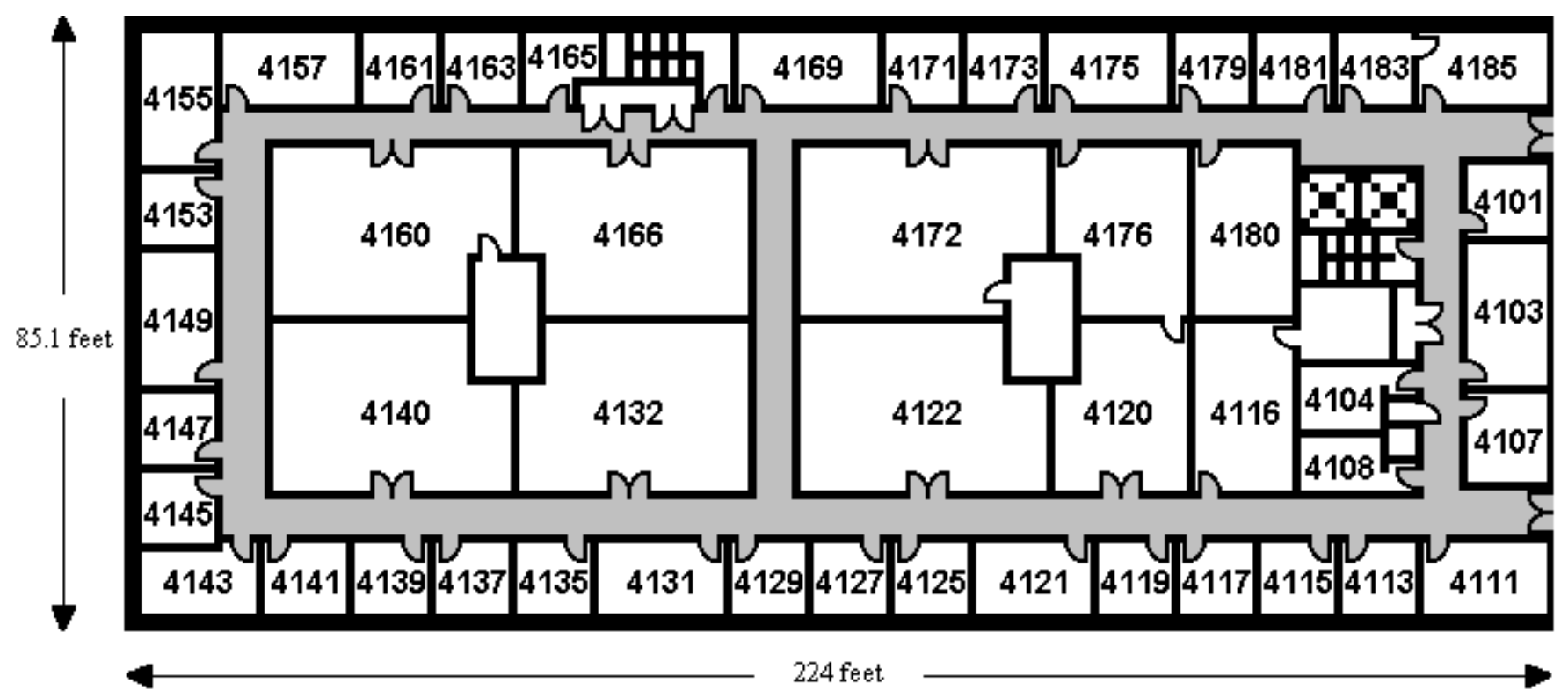

Fig. 3. Floor plan for the first testbed. Readings were collected in the corridors and inside the rooms.

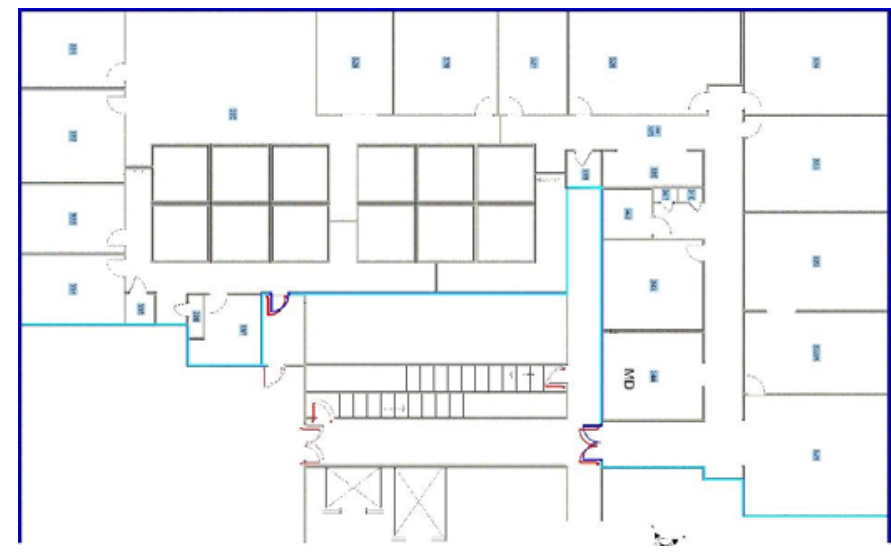

Fig. 4. Floor plan of the office space where the second experiment was conducted. Readings were collected in the corridors and inside the rooms.

The figures also show that there is an optimal value for the parameter $N$ around $N=6$. As $N$ is increased beyond this value, more noise is introduced due to adding locations that are far away from the correct location.

2) Time-averaging Technique: Figures 7 and 8 show the effect of increasing the parameter $W$ (size of the averaging window) on the performance of the time-averaging technique for the two testbeds. The figures show that the larger the averaging window, the better the accuracy. The performance of the $\mathrm{Ho}$ rus system is enhanced by more than $24 \%$ for the first testbed and more than $15 \%$ for the second testbed for $W=10$. Note that the higher the value of $W$, the slower the system to react to a fast changes in the user location, e.g. a fast moving tracked object. Therefore, we have a tradeoff between the accuracy we can obtain and the system responsiveness.

\section{Discussion}

The results show that the continuous-space estimation techniques enhance the accuracy significantly. The two techniques can be combined together to further enhance the accuracy. The output of the Center of Mass technique can be used as an input to the Time Averaging technique to obtain a better estimate compared to using the discrete space estimator.

\section{RELATED WORK}

Radio-map based WLAN location determination systems can be categorized into two broad categories: deterministic techniques and probabilistic techniques. Deterministic techniques [6-9] represent the signal strength of an access point at a location by a scalar value, for example, the mean value, and use non-probabilistic approaches to estimate the user location. For example, in the Radar system [6,7] the authors use nearest neighborhood techniques to infer the user location. On the other hand, probabilistic techniques [1-4, 10-14] store information about the signal strength distributions from the access points in the radio map and use probabilistic techniques to estimate the user location. For example, the Nibble system $[10,11]$ uses a Bayesian Network approach to estimate the user location.

Our proposed continuous space estimation techniques can be applied to the above systems to further enhance their accuracy.

\section{CONCLUSIONS AND FUTURE WORK}

In this paper, we described two techniques for allowing continuous-space estimation: the Center of Mass technique and the Time Averaging technique. Using the Center of Mass technique, the performance of the Horus system is enhanced by more than $13 \%$ for the first testbed and more than $6 \%$ for the second testbed compared to the basic technique. The TimeAveraging technique enhances the performance of the Horus system by more than $24 \%$ for the first testbed and more than $15 \%$ for the second testbed. The two techniques are independent and can be applied together. Moreover, both techniques are general and may be applied to other WLAN location determination systems. 


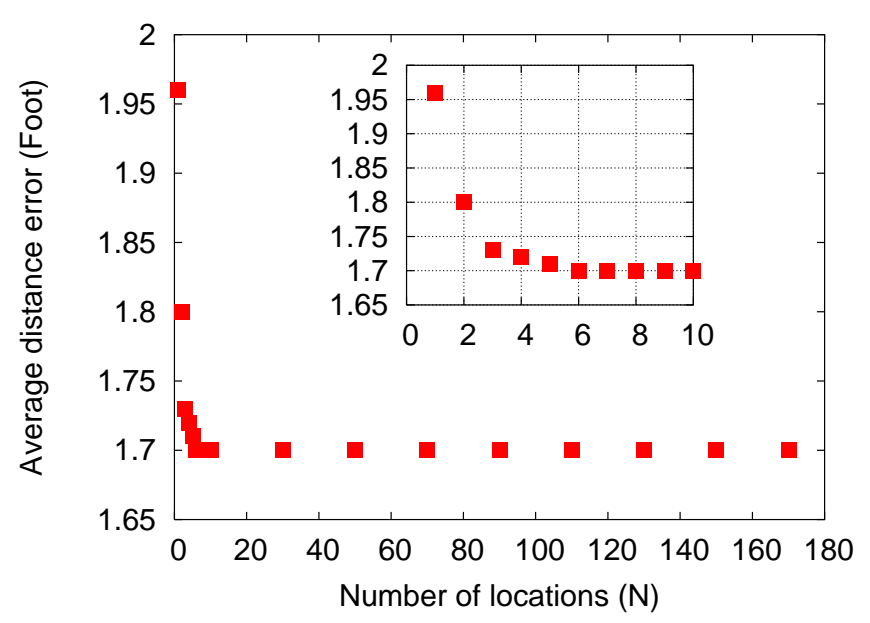

Fig. 5. Average distance error using the Center of Mass technique for the first testbed. The sub-figure shows the same curve for $N=0-10$.

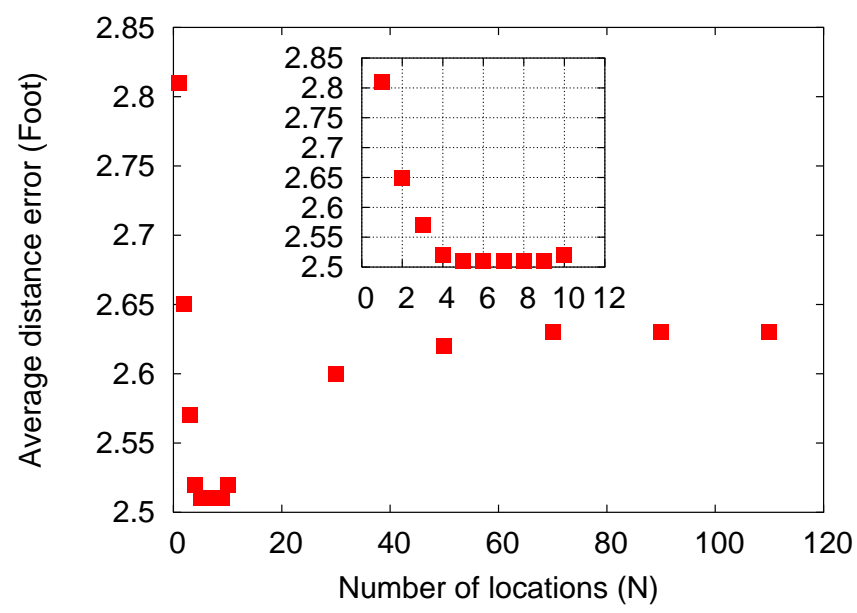

Fig. 6. Average distance error using the Center of Mass technique for the second testbed. The sub-figure shows the same curve for $N=0-12$.

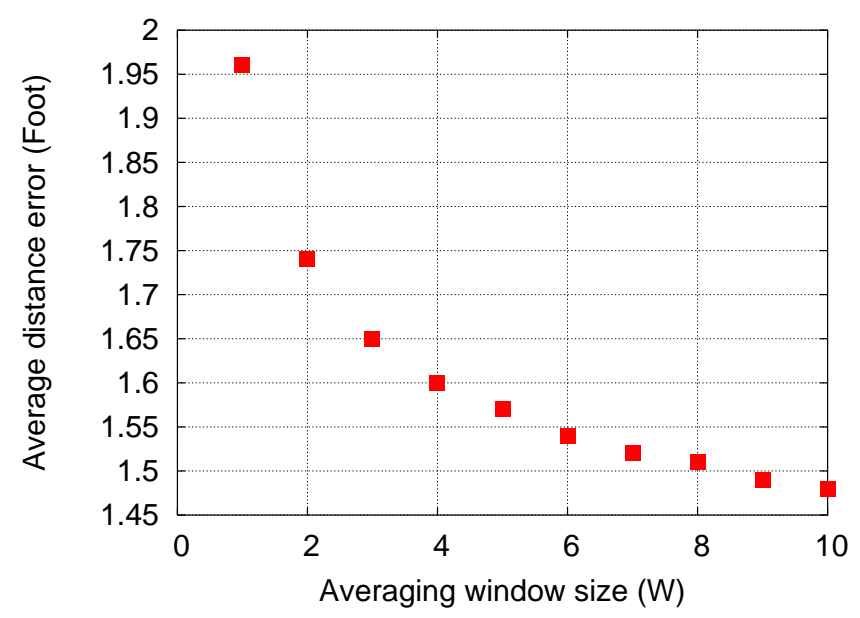

Fig. 7. Average distance error using the time-averaging technique for the first testbed.

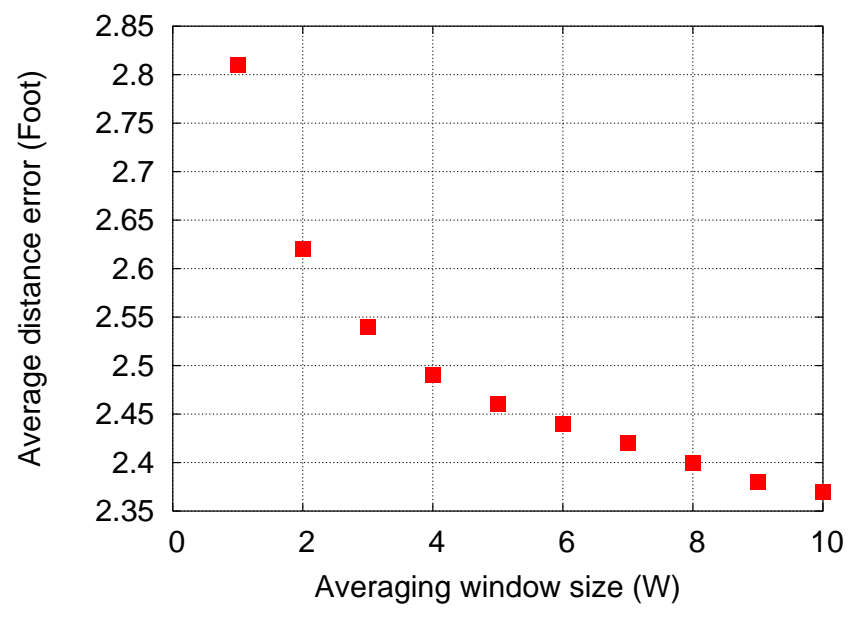

Fig. 8. Average distance error using the time-averaging technique for the second testbed.

We are currently working on techniques to dynamically change the system parameters. For example, the size of the averaging window can be changed dynamically to match the speed of the object being tracked.

\section{ACKNOWLEDGMENT}

This work was supported in part by the Maryland Information and Network Dynamics (MIND) Laboratory, its Founding Partner Fujitsu Laboratories of America, and by the Department of Defense through a University of Maryland Institute for Advanced Computer Studies (UMIACS) contract.

\section{REFERENCES}

[1] M. Youssef, A. Agrawala, and A. U. Shankar, "WLAN Location Determination via Clustering and Probability Distributions," in IEEE PerCom 2003, March 2003.

[2] Moustafa Youssef and Ashok Agrawala, "Handling samples correlation in the horus system," in IEEE Infocom, March 2004.

[3] M. Youssef and A. Agrawala, "Small-Scale Compensation for WLAN Location Determination Systems," in IEEE WCNC 2003, March 2003.

[4] M. Youssef, A. Agrawala, A. U. Shankar, and Sam H. Noh, "A Probabilistic Clustering-Based Indoor Location Determination System," Tech. Rep. UMIACS-TR 2002-30 and CS-TR 4350, University of Maryland, College Park, March 2002, http://www.cs.umd.edu/Library/TRs/.

[5] M. Youssef and A. Agrawala, "On the Optimality of WLAN Location Determination Systems," in Communication Networks and Distributed Systems Modeling and Simulation Conference, January 2004.

[6] P. Bahl and V. N. Padmanabhan, "RADAR: An In-Building RF-based User Location and Tracking System," in IEEE Infocom 2000, March 2000, vol. 2, pp. 775-784.

[7] P. Bahl, V. N. Padmanabhan, and A. Balachandran, "Enhancements to the RADAR User Location and Tracking System," Tech. Rep. MSR-TR-0012, Microsoft Research, February 2000.

[8] A. Smailagic, D. P. Siewiorek, J. Anhalt, D. Kogan, and Y. Wang, "Location Sensing and Privacy in a Context Aware Computing Environment," Pervasive Computing, 2001.

[9] P. Krishnan, A.S. Krishnakumar, Wen-Hua Ju, Colin Mallows, and Sachin Ganu, "A system for lease: Location estimation assisted by stationary emitters for indoor rf wireless networks," in IEEE Infocom, March 2004.

[10] P. Castro, P. Chiu, T. Kremenek, and R. Muntz, "A Probabilistic Location Service for Wireless Network Environments," Ubiquitous Computing 2001, September 2001.

[11] P. Castro and R. Muntz, "Managing Context for Smart Spaces," IEEE Personal Communications, OCTOBER 2000.

[12] T. Roos, P. Myllymaki, H. Tirri, P. Misikangas, and J. Sievanen, "A Probabilistic Approach to WLAN User Location Estimation," International Journal of Wireless Information Networks, vol. 9, no. 3, July 2002. 
[13] T. Roos, P. Myllymaki, and H. Tirri, "A Statistical Modeling Approach to Location Estimation," IEEE Transactions on Mobile Computing, vol. 1, no. 1, pp. 59-69, January-March 2002.

[14] A. M. Ladd, K. Bekris, A. Rudys, G. Marceau, L. E. Kavraki, and D. S. Wallach, "Robotics-Based Location Sensing using Wireless Ethernet," in 8th ACM MOBICOM, Atlanta, GA, September 2002.

[15] S. Ganu, A.S.Krishnakumar, and P.Krishnan, "Infrastructure-based Location Estimation in WLAN Networks," in IEEE Wireless Communications and Networking Conference, March 2004.

[16] K. Kaemarungsi and P. Krishnamurthy, "Modeling of Indoor Positioning Systems Based on Location Fingerprinting," in IEEE Infocom, March 2004.

[17] Y. Gwon, R. Jain, and T. Kawahara, "Robust Indoor Location Estimation of Stationary and Mobile Users," in IEEE Infocom, March 2004.

[18] G. Chen and D. Kotz, "A Survey of Context-Aware Mobile Computing Research,” Tech. Rep. Dartmouth Computer Science Technical Report TR2000-381, 2000.

[19] "http://www.cs.umd.edu/users/moustafa/Downloads.html," .

[20] "http://www.orinocowireless.com," . 